\title{
THE NEURAL CORRELATES OF THEORY OF MIND AND THEIR ROLE DURING EMPATHY AND THE GAME OF CHESS: A FUNCTIONAL MAGNETIC RESONANCE IMAGING STUDY
}

\author{
JOANNE L. POWELL, ${ }^{a, b *}$ DAVIDE GROSSI, ${ }^{c}$ \\ RHIANNON CORCORAN, ${ }^{d}$ FERNAND GOBET ${ }^{e}$ AND \\ MARTA GARCÍA-FIÑANA \\ ${ }^{a}$ Magnetic Resonance and Image Analysis Research Centre \\ (MARIARC), University of Liverpool, UK \\ ${ }^{\mathrm{b}}$ Department of Psychology, Edge Hill University, UK \\ ${ }^{c}$ Department of Computer Science, University of Liverpool, UK \\ ${ }^{\mathrm{d}}$ Institute of Psychology, Health and Society, University of \\ Liverpool, UK \\ e Department of Psychological Sciences, University of Liverpool, UK \\ ${ }^{\mathrm{f}}$ Department for Biostatistics, University of Liverpool, UK
}

\begin{abstract}
Chess involves the capacity to reason iteratively about potential intentional choices of an opponent and therefore involves high levels of explicit theory of mind [ToM] (i.e. ability to infer mental states of others) alongside clear, strategic rule-based decision-making. Functional magnetic resonance imaging was used on 12 healthy male novice chess players to identify cortical regions associated with chess, ToM and empathizing. The blood-oxygenationlevel-dependent (BOLD) response for chess and empathizing tasks was extracted from each ToM region. Results showed neural overlap between ToM, chess and empathizing tasks in right-hemisphere temporo-parietal junction (TPJ) [BA40], left-hemisphere superior temporal gyrus [BA22] and posterior cingulate gyrus [BA23/31]. TPJ is suggested to underlie the capacity to reason iteratively about another's internal state in a range of tasks. Areas activated by ToM and empathy included right-hemisphere orbitofrontal cortex and bilateral middle temporal gyrus: areas that become active when there is need to inhibit one's own experience when considering the internal state of another and for visual evaluation of action rationality. Results support previous findings, that ToM recruits a neural network with each region sub-serving a supporting role depending on the nature of the task itself. In contrast, a network of cortical regions primarily located within right- and left-hemisphere medial-frontal and parietal cortex, outside the internal representational network, was selectively recruited during the chess task. We hypothesize that in our cohort of novice chess players the strategy was to employ an iterative thinking pattern which in part involved mentalizing processes
\end{abstract}

\footnotetext{
*Correspondence to: Joanne L. Powell, Department of Psychology, Edge Hill University, Ormskirk, Lancashire L39 4QP, UK.

E-mail address: joanne.powell@edgehill.ac.uk (J. L. Powell) Abbreviations: BOLD, blood-oxygen-level-dependent; FDR, false discover rate; fMRI, functional magnetic resonance imaging; HRF hemodynamic response function; IMT, imposing memory task; IPL, inferior parietal lobule; PET, positron emission tomography; PFC, prefrontal cortex; ToM, theory of mind; TPJ, temporo-parietal junction.
}

and recruited core ToM-related regions. (c) 2017 The Authors. Published by Elsevier Ltd on behalf of IBRO. This is an open access article under the CC BY-NC-ND license (http://creativecommons.org/licenses/by-nc-nd/4.0/).

Key words: theory of mind, chess, empathizing, temporoparietal junction, fMRI.

\section{INTRODUCTION}

Theory of Mind (ToM), also referred to as intentionality and mentalizing (Frith and Frith, 1999; Völlm et al., 2006), is the ability to infer the intentions, beliefs or mental states of others in order to explain and predict behavior (Powell et al., 2010, 2014; Stiller and Dunbar, 2007). Neuroimaging literature suggests that ToM is associated with a distributed network of cortical regions (reviewed by Carrington and Bailey, 2009, and Lieberman, 2007). Regions most commonly reported include, medial frontal gyrus [MFG: BA8/9], inferior frontal gyrus [IFG: BA47], ventromedial and ventrolateral prefrontal cortex (PFC) [both of which partially overlap orbital PFC and include BA11], temporoparietal junction [TPJ: BA40], superior temporal sulcus [STS: BA21] and precuneus [BA7] (see Powell et al., 2014). The extent to which these different regions are involved depends on the nature of the social cognitive task being used (Lieberman, 2007; Powell et al., 2014). A 'core-network' for ToM has been proposed, which includes medial PFC (mPFC) and bilateral TPJ (Amodio and Frith, 2006; Frith and Frith, 2006; Mitchell, 2009; Schurz et al., 2014). These regions are consistently engaged whenever we reason about the mental states of others, regardless of task and stimuli (Schurz et al., 2014). Empathizing, while important for inferring the emotional states of others and for successful social interaction (Baron-Cohen et al., 2001; BaronCohen and Wheelwright, 2004), is different to ToM: it is the capacity to comprehend, infer, judge and share the emotional experiences of another (Gallese, 2003). Using functional magnetic resonance imaging (fMRI), Völlm et al. (2006) showed that ToM and empathy are associated with overlapping but distinct neural networks.

Knowing how another person thinks and 'thinks you think' etc. is critical to predicting behavior in strategic interaction games (Camerer et al., 2005). Iterated strategic thinking consumes working memory and requires the ability to metaphorically 'put ourselves in another player's 
mind' (Camerer et al., 2005). It seems then that ToM and strategic interactions both require similar cognitive processes. However, while strategic interactions require iterative reasoning about another's mind state from a purely strategic perspective (where the potential moves are predefined and based on a set of structured rules, as is the case during strategic interactions), ToM requires iterative reasoning about another's mind state within social contexts (where rules are much less well defined, ambiguity is pervasive and decisions are based on prior experience and expectations which might be culturally and contextually embedded).

Studies exploring strategic interactions during gaming situations (e.g. the Prisoners Dilemma, Dictator and Ultimatum games) suggest that the ability to infer the mental states of one's opponent is beneficial to making the best choice (McCabe et al., 2001; Sally and Hill, 2006; Behrens et al., 2009). The game of chess involves facets of high-level cognition and problem-solving abilities (Atherton et al., 2003) and, at least in novice chess players, the capacity to reason iteratively about the potential moves of the opponent. It provides a simple environment, using chess pieces that have a finite number of moves but through which an immense number of possibilities can be generated $\left(2^{143}\right.$, see de Groot and Gobet, 1996). Games like the prisoners' dilemma and ultimatum game have a social motive or empathizing component which occurs during a strategic interaction (for example, altruism, fairness, reciprocity, and cooperation), which is not present in the game of chess. The strategies of novice chess players differ from those employed by expert chess players. Expert chess players are thought to automatically call to memory perceptual patterns of game play (known as 'chunks') when perceiving familiar positions (Chase and Simon, 1973; Gobet, 1998) and use them for carrying out look-ahead search (Gobet, 1997), whereas novice chess players proceed only using an iterative strategic thinking pattern. This would suggest that in novice chess players there is a large degree of neural overlap when the chess players are considering potential moves on a chess board and tasks that involve assigning mental states to others (i.e. ToM-related tasks), and this is the primary aim of the present study.

To explore the underlying mechanisms of social cognition, some neurocognitive studies have used a game theory approach (e.g. King-Casas et al., 2008; Behrens et al., 2009; Tayama et al., 2012). During games that involve strategic interactions, fMRI and positron emission tomography (PET) studies show that playing humans versus computers activates ToM areas (Gallagher et al., 2002; Camerer, 2009). This suggests that strategic interactions during games that require an iterative component are not purely based on a computational task, but do require some degree of social cognition such as the understanding that the other player holds a mind state that is different from one's own. Few studies have looked at the neural correlates of chess, but those that have, show neural regions associated with chess in bilateral frontal lobes, parietal lobes and occipital lobes (Atherton et al., 2003; Campitelli et al., 2005). This study will investigate the neural network associated with ToM, using a well-established ToM task (Völlm et al., 2006) and identify whether a significant blood-oxygenationlevel-dependent (BOLD) response for the processing of a chess task and empathizing task is significant within those pre-defined regions identified using the ToM task.

\section{METHODS AND MATERIALS}

\section{Participants}

Participants were 12 males, all right-handed, aged 2058 years (mean age $=36.42 \mathrm{yrs}, \quad S D=13.91 \mathrm{yrs}$ ). All are chess players with different levels of experience, who know the rules of chess and have at some point belonged to a chess club. Years playing chess ranged from 4 to 48 years (mean years playing chess $=26.33 \mathrm{yrs}, \mathrm{SD}=13.75 \mathrm{yrs}$ ). Participants learned to play chess between 6 and 17 years of age (mean age learned to play chess $=10.17 \mathrm{yrs}, \quad S D=3.22 \mathrm{yrs}$ ). Intensity of play refers to the frequency of chess play. For the individual to qualify as having played chess with intense periods of play, they must have played regularly at a chess club for a period of 6 months and report playing the game at least three times a week. Participants were recruited from University of Liverpool and Merseyside Chess clubs. All participants gave signed informed consent, and the study had the approval of the local research ethics committee.

\section{Neuropsychological protocol}

Participants completed an imposing memory task (IMT) used previously (Lewis et al., 2011; Powell et al., 2010, 2012a, 2014). The IMT provides a measure of the individuals' ability to infer the mental states of others. The task involves reading five short stories twice, each approximately 200 words in length. Stories depict a social situation (e.g. an employee trying to decipher, from a work colleague, whether a different colleague might be interested in him). Following each story, the participant answers a set of 20 true/false questions containing an equal number of intentionality questions and factual (short-term memory) questions to distinguish between intentionality capacity and ability to remember factual information. Intentionality questions require complex mentalizing about a character's perspective within a social situation. The questions vary in complexity and require the individual to represent the mind states of others, up to and including level six intentionality and short-term memory. Further details of the IMT, including an example story and questions, as well as the equation for calculating intentionality and short-term memory scores, are provided in Powell et al. (2014).

\section{fMRI activation tasks}

Participants completed three experimental tasks in the scanner: a Theory of Mind (ToM) task, an empathizing task and a chess task. Task stimuli were presented using 'Presentation' software (https://nbs.neuro-bs.com). All stimuli were presented in blocks. The ToM task and empathizing task have been used previously (Brunet et al., 2000; Völlm et al., 2006). With these two tasks, the participant is first presented with a short comic strip 
consisting of three images. A further two images are then presented below. The participant is asked to choose which of the two further images complete the story emerging from the first three images the best. The chess task was designed to match as closely as possible the design of the ToM and the empathizing tasks. The participants are presented with a strip of images of a chess board depicting a game in progress. Further two images are presented below this and the participant is asked to decide which of the two depicted moves in the further images would make the most sense within the sequential four move series. In all conditions, participants pressed the left key for "Image 1" and the right key for "Image 2".

Theory of mind and empathizing task designs. In the ToM task, the experimental condition involved inferring the mental state of the character in the story. The participant is asked "What will the character do next?" In this condition, no social interactions of emotional situations are depicted and therefore there is only one character in the story. In the empathizing task, the participant is asked, "What will make the main character feel better?" The participant must empathize with the protagonist in the story in order to make the correct answer. Two characters are depicted in the empathizing condition. In the control condition the participant is presented with a story that requires only comprehension of the physical causalities of the protagonist in the story and must decide "What is most likely to happen next?" In order to match for the correct number of characters in the story and its complexity, the control condition for the ToM task included only one character while the control condition for the empathizing task included two characters. Thus in total there were four categories of story: 1. Theory of Mind ('ToM'); 2. Empathizing ('Empathy'); 3. Physical causality with one character ('Physical 1'); and 4. Physical causality with two characters ('Physical 2'). There are ten different stories per condition. Each story is presented twice with each block consisting of five different stories resulting in a total of four blocks per condition. A short question introducing the block is shown for $6 \mathrm{~s}$. The comic strip depicting the story is shown in the upper half of the screen for $6 \mathrm{~s}$ and the two pictures showing the possible outcomes of the scenario are imposed on the bottom half of the screen for a further $6 \mathrm{~s}$, during which the participant must respond with their correct answer.

Chess task design. In the experimental chess task ('chess'), the participants had to decide "What move would you make?" This scenario depends on the participant following the progression of a game as it develops and deciding which out of two options, depicting the next move, they would make next. The chess games were chosen at random from a database of previous chess masters'/grandmasters' games (http:// www.chess.com/games/) with the starting point randomly selected from the game. An example of the chess task and control condition is shown in Fig. 1. Participants must choose between two images to indicate the move that they would make next if they were playing the game. One image is the move taken by the chess master/grandmaster during the chess game (correct response) and the second image shows a move of a different chess piece (incorrect response). In the control condition ('ball') the participant has to decide "Where will the ball land next?" The participants are instructed that there is only one rule in the ball scenario which is that the ball can only move from one side of the board to the opposite site, i.e. either left to right or top to bottom, but never, for example, from the top of the board to the left or right side or from the right side of the board to the top or bottom of the board. The ball is shown in yellow to match the yellow presented in the chess task condition. This scenario depends only on physical causality and requires the participant to follow where the ball has moved from or to. The chess pieces are also randomly dispersed on the board. The placement of the pieces in the middle of the squares was avoided, as suggested by Atherton et al. (2003), to avoid participants considering possible moves. A total of twelve different scenarios per condition were used. Each block consists of three different scenarios resulting in four blocks per condition. At the beginning of each block participants are presented with a short question introducing the block: shown for $6 \mathrm{~s}$. The block consists of two different games. The strip depicting the game or ball in progress is shown in the upper half of the screen for $12 \mathrm{~s}$ and the two pictures showing the possible outcomes of the scenario are imposed on the bottom half of the screen for a further $12 \mathrm{~s}$

\section{MRI data acquisition}

MR images were obtained using a 3 Tesla Siemens Trio (Siemens Medical, Erlangen, Germany) scanner with an eight-channel head coil. High-resolution T1-weighted anatomical images were acquired sagitally using the following parameters: TE $5.57 \mathrm{~ms}$, TR $2040 \mathrm{~ms}$, flip angle $8^{\circ}, \mathrm{FOV}=256,176$ slices, voxel size $1 \times 1 \times 1 \mathrm{~mm}^{3}$. Functional images were obtained using a $\mathrm{T}_{2}$-weighted gradient echo EPI sequence with the following parameters: $\mathrm{TE}=35 \mathrm{~ms}$; $\mathrm{TR}=3000 \mathrm{~ms}$; flip angle $90^{\circ}$, slice thickness $3 \mathrm{~mm}, 0.3 \mathrm{~mm}$ gap, matrix $64 \times 64$, FOV $=192 \mathrm{~mm}$; in-plane resolution $3 \times 3 \mathrm{~mm}$, 43 slices. Forty-three axial slices oriented parallel to the AC-PC line covering the whole brain were taken. Foam padding was used to prevent head movements during the scan.

\section{MRI data analysis}

The Statistical Parametric Mapping software package (SPM8, available at Welcome Department of Cognitive Neurology, London, UK, http://www.fil.ion.ucl.ac.uk/spm) was used for realignment, normalization and smoothing and statistical analysis to create statistical parametric maps of significant regional BOLD response changes (Friston et al., 1995a,b). Prior to pre-processing the first two images of each experimental run were discarded. The image time series was first realigned to the first image (of the remaining time series). Sinc interpolation was used in the transformation. The $T_{1}$-weighted image for each individual was coregistered to their mean functional image created from the realigned images. The 

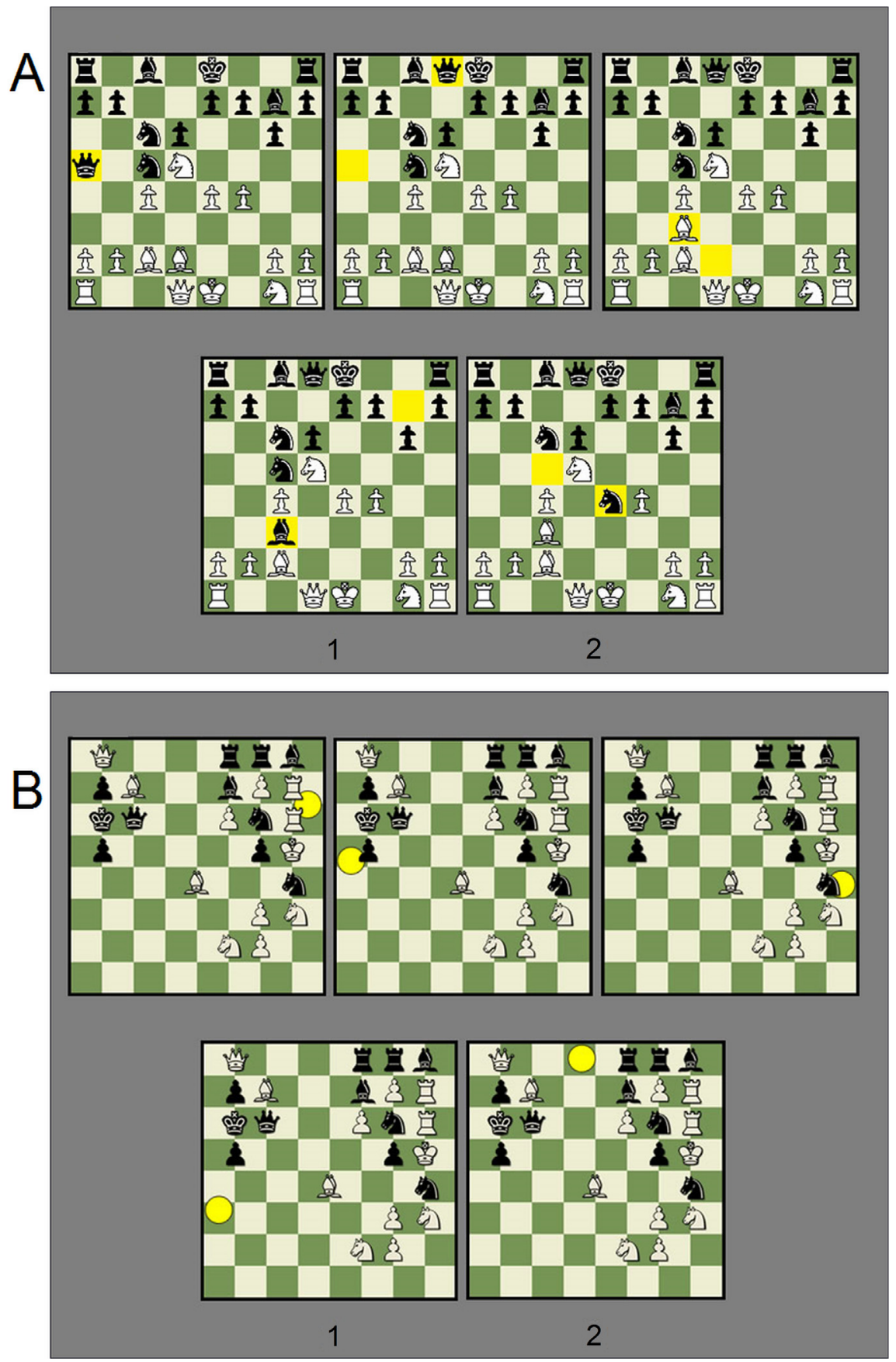

Fig. 1. Examples of a chess task scenario $(A)$ and a control task scenario $(B)$ for the fMRI chess task. In image $(A)$ the chess pieces on yellow squares indicate the piece that is to be moved (top row - first image) or has been moved (top row - second and third images) and the block yellow square indicates where the piece has moved from. The participant must decide which move they would take next out of image 1 or image 2 (bottom row). In this example, image 1 is the correct response. This game is taken from the 1998 game between Jan Timman and Rui Damaso (http:// www.chess.com/games/view?id=861309\#), after moving 10.Nd5. Image B shows the control condition; the ball can only move from one side of the chess board to the opposite side. Participants must decide where the ball will land next. In this example image 1 is the correct response.

structural image was segmented in native space using the VBM toolbox (VBM8) http://dbm.neuro.uni-jena. de/software. The resulting gray matter segment was then normalized to the a priori Montreal Neurological Institute (MNI) gray matter template supplied by SPM8. The resulting parameters were then applied to normalize the functional images and $T_{1}$-weighted images into MNI space (Friston et al., 1995a). The resulting pixel size in standard stereotaxic coordinates was $2 \times 2 \mathrm{~mm}$, with an interplane distance of $2 \mathrm{~mm}$. The normalized images were finally smoothed with an isotropic six FWHM Gaussian kernel prior to statistical analysis.

\section{Statistical analysis}

The time series was filtered with a high-pass filter of $128 \mathrm{~s}$ to remove subject-specific low-frequency drifts. The experimental conditions (e.g. 'ToM' relative to 'Physical 1') were modeled using a boxcar function convolved with a hemodynamic response function (HRF) (Friston et al., 1994) in the context of the general linear model employed by SPM8. Specifically, BOLD signal changes to the 'ToM', 'Empathy' or 'Chess' respectively relative to 'Physical 1', 'Physical 2' or 'Ball' were modeled. This resulted in three contrast images per participant. Group results for each task were generated by importing individual contrast images into a second-level analysis. A full-factorial model was employed to establish the overall pattern of activation for each task. The variables, age, weighted chess level, intentionality score and short-term memory score were entered into the model as covariates.

A 'weighted chess level' was given to each participant to reflect their expertise as a chess player. The weighted chess level is based on the intensity at which the individual has played chess and the number of correct responses they gave during the fMRI chess task (i.e. the number of times they agreed with the option chosen by a Grand Master referred to as 'correct chess answers'). This was calculated using the following formula:

\section{Weighted chess level}

\section{$=($ intensity $\times$ correct chess answers $)$}

The statistical parametric maps were interpreted after applying a false discover rate (FDR) correction with $P<0.05$. Only clusters of at least $k \geq 10$ voxels were selected for inclusion in further analysis.

Second-level analysis revealed 12 significant clusters in which activation was significantly greater for the 
contrast 'ToM'-'Physical 1' across all participants. A mask was then created for each of these cluster regions using the toolbox MarsBaR (http://marsbar.sourceforge.net/) in SPM8. Parameter estimates for all voxels within each cluster region were obtained and these parameter estimates were then averaged across the cluster region for the three contrasts: 'ToM'-'Physical 1'; 'Empathy''Physical 2'; and 'Chess'-'Ball', using the secondlevel contrast image. Regions of significant association were identified using the Wake Forest University Pickatlas (http://fmri.wfubmc.edu/cms/software\#Pick Atlas; Maldjian et al., 2004) using Talairach coordinates of the most significant voxel $(x, y, z \mathrm{~mm})$.

\section{RESULTS}

Descriptive statistics for the chess variables used in this study, and IMT scores can be found in Table 1. Weighted chess level ranged from 5 to 36 (mean $=16.4, \mathrm{SD}=10.5$ ).

Neural responses associated with the processing of ToM stimuli (contrast 'ToM' - 'Physical 1'), empathy stimuli (contrast 'Empathy'-'Physical 2') and chess stimuli (contrast 'Chess'-'Ball') can be seen across the whole brain (in left and right hemispheres) in Fig. 2 (panels A, B and C respectively). The contrast 'ToM' 'Physical 1' yielded a total of twelve areas of activation, which are shown in Appendix A and Fig. 3. Contrast values for comparisons 'ToM'-'Physical 1'; 'Empathy'-'P hysical 2'; and 'Chess'-'Ball' were extracted across these twelve regions (shown in Appendix A). Areas that were significant for all three contrast conditions included left hemisphere superior temporal gyrus [BA22 (Fig. 3, region 6)] and posterior cingulate gyrus [BA23/31 (Fig. 3, region 5)] and right hemisphere temporoparietal junction [BA40 (Fig. 3, region 4)]. Results showed significant activation for the contrast 'ToM'-'Physical 1' and 'Empathy'-'Physical 2' in right hemisphere medial frontal gyrus/orbitofrontal cortex [BA11 (Fig. 3, region 3)] and bilateral medial temporal gyrus [BA21 (Fig. 3, regions 1 and 2)]. Significant activation for the contrasts 'ToM'-'Physical 1' and 'Chess'-'Ball' were found in six of the twelve regions: left hemisphere cuneus [BA18 (Fig. 3, region 11)], fusiform gyrus [BA37 (Fig. 3, region 7)], inferior frontal gyrus [BA45 (Fig. 3, region 12)], and right hemisphere pyramis/cerebellum posterior lobe (Fig. 3, region 8), lingual gyrus [BA18 (Fig. 3, region 9)], and middle occipital gyrus [BA19 (Fig. 3, region 10)].

Areas of activation for the contrast conditions 'Empa thy'-'Physical 2' and 'Chess'-'Ball' (tested across the whole brain) are shown in Appendix B. The contrast 'Empathy' - 'Physical 1' yielded twelve areas of activation in bilateral anterior cingulate gyrus [BA24], and middle temporal gyrus [BA21], left hemisphere superior [BA9] and inferior [BA45] frontal gyrus, cingulate gyrus [BA24] and uvula/cerebellum posterior lobe, and right hemisphere precuneus [BA7], inferior parietal lobule [BA40], temporoparietal junction [BA40] and medial frontal gyrus [BA11]. The contrast 'Chess'-' Ball' tested across the whole brain yielded ten areas of activation in left hemisphere precuneus [BA7], caudate tail, precentral gyrus [BA6], middle frontal gyrus [BA6 and BA46], posterior cingulate gyrus [BA23] and culmen/cerebellum anterior lobe and right hemisphere temporoparietal junction [BA40], middle frontal gyrus [BA9] and declive/cerebellum posterior lobe.

The effect of intentionality score, short-term memory score, age and weighted-chess level was tested for within the regions of activation generated from each of the three contrasts (i.e. 'ToM'-'Physical 1'; 'Empathy'-'P hysical 2'; and 'Chess'-'Ball'). No significant results were found following correction for multiple comparisons (FDR, $P<0.05)$

\section{DISCUSSION}

Findings from the present study show a distributed neural network involved in ToM, consistent with previous literature (Lieberman, 2007; Carrington and Bailey, 2009), which shows a role for superior temporal gyrus [BA22] (Völlm et al., 2006) and superior temporal sulcus [STS: BA21/22] (Allison et al., 2000; Frith and Frith, 2003; Völlm et al., 2006; Enrici et al., 2011), temporoparietal junction [TPJ: BA40] (Gallagher et al., 2000; Völlm et al., 2006; Gobbini et al., 2007; Enrici et al., 2011) and orbital PFC [OPFC] (Brunet et al., 2000; Powell et al., 2010, 2014; Spreng et al., 2009; Völlm et al., 2006) in ToM processing. The study also showed a role for cuneus [BA18], fusiform gyrus [BA37] and right cerebellum associated with the ToM task, consistent with the findings of Völlm et al. (2006).

A total of twelve different areas of activation were found in relation to ToM processing, none of which were unique to ToM itself. When the twelve regions were used as pre-defined regions of interest all three tasks were associated with significant neural activation in three of the twelve regions: left hemisphere superior temporal gyrus [BA22] and posterior cingulate gyrus [BA23/31] and right hemisphere TPJ [BA40]. Previous literature supports the role of each of these regions in

Table 1. Mean scores, standard deviations and minimum and maximum scores for age, weighted chess level (and the chess variables used to calculate this score), years of playing chess, intentionality and short-term memory score (obtained from the IMT)

\begin{tabular}{llll}
\hline Variables & Mean & SD & Min. \\
\hline Age (in years) & 36.4 & 13.9 & 20 \\
Weighted Chess level & 16.4 & 10.5 & 5 \\
Years playing chess & 26.3 & 13.8 & 36 \\
Intensity of chess play & 2.2 & 1.1 & 4 \\
Score on fMRI chess task & 7.3 & 1.5 & 1 \\
Intentionality (IMT) & 4.6 & 0.3 & 5 \\
Short-term Memory (IMT) & 5.0 & 0.2 & 4.1 \\
\hline
\end{tabular}




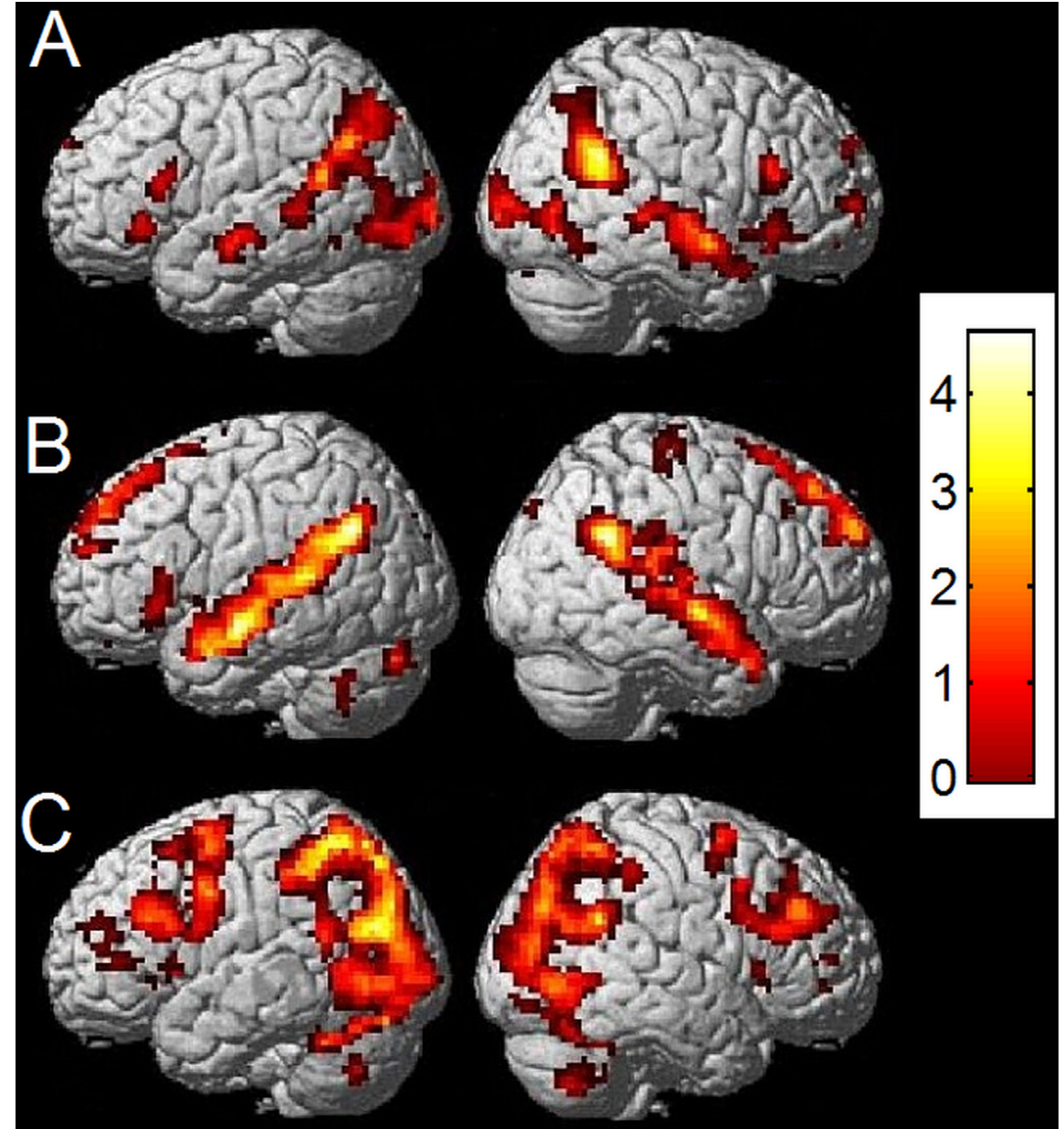

Fig. 2. Areas of activation associated with the contrasts: 'ToM' - 'Physical 1' (panel A); 'Empathy' - 'Physical 2' (panel B); and 'Chess' - 'Ball' (panel C), performed across whole brain. Significant regions of activation are displayed laterally on a cortical surface rendered brain on the left hemisphere and right hemisphere. Displayed results are significant at $P<0.05$ with the false discover rate (FDR) correction for multiple comparisons.

ToM (Völlm et al., 2006; for review see Lieberman, 2007), chess (Campitelli et al., 2005) and empathy (for review see Bernhardt and Singer, 2012) tasks. ToM and empathy tasks were associated with significant activation in a further three regions: right hemisphere medial frontal gyrus/orbitofrontal cortex [BA11] and bilateral medial temporal gyrus [BA21]; neural activation associated with the chess task was not found in these three regions. Middle and superior [BA21/22] temporal gyri are consistently reported in ToM studies (Frith and Frith, 2003; Lieberman, 2007), which supports the findings in the present study. Middle temporal gyrus is bounded dorsally by superior temporal sulcus which sits ventrally to the superior temporal gyrus. Posterior superior temporal gyrus [BA22] is thought to be particularly sensitive to biological motion (Allison et al., 2000), which is an externally focused process that does not require consideration of the other's internal mental state. Other research indicates a role for the superior temporal gyrus more generally in movement kinematics not only perception of human movement (Jastorff and Orban, 2009), which might explain its role in all three tasks used in the present study.
In addition to its role in ToM (e.g. Frith and Frith, 2003; Völlm et al., 2006; Lieberman, 2007), middle temporal gyrus [BA21] has been shown to be sensitive to the visual evaluation of action rationality (Jastorff et al., 2011). Jastorff et al. (2011) found that the medial temporal gyrus is sensitive to weighing up predictions about external reality made from an internal working model. In their study, when the expected pattern of rational goal attainment was violated, there was an increase in neural activity within medial temporal gyrus. In this respect, it is the action kinematics of the characters (in the ToM and empathizing task) and chess pieces (in the chess task) that recruits neural activation in superior temporal gyrus, but it is the rationality of the characters in the ToM and empathizing tasks, i.e. the contextual elements in which the characters are constrained by, that leads to activation of middle temporal gyrus. While rationality of the opponent's move during the chess task might be considered, the role of the medial temporal gyrus might be contingent on the relationship between goal directed biological action and its relevant environmental constraints.

Temporo-parietal junction [TPJ; BA40: which consists of supramarginal gyrus within inferior parietal lobule (IPL) and a caudal portion of the superior temporal gyrus], is a key neural region in ToM tasks (Saxe and Wexler, 2005). While situated anatomically close to superior temporal sulcus, the TPJ is considered to be distinct from this region when it comes to its role in ToM tasks (Saxe et al., 2004). This region is thought to be particularly important for reasoning about the mental states of others, and is robustly reported in imaging studies that involve 'false-belief' tasks (Saxe and Kanwisher, 2003). This region is also thought to be important for distinguishing between self and other (Lawrence et al., 2006). The empathy task used in the present study involved imagining the affective state of another and comprises affective sharing, self-awareness and self-other distinction (Bernhardt and Singer, 2012). It is not, therefore, surprising that TPJ significantly activated by the ToM task, was also significantly associated with the empathy task. Neural activation associated with the chess task was also found in TPJ. One explanation for this association might be that TPJ was playing a similar role during the chess task, as participants reasoned about the potential mental states of the opponent. In all tasks, it was right hemisphere TPJ that was activated, which Saxe and Wexler (2005) have shown to be important in the attribution of mental states. Another explana- 
tion for the role of TPJ during the chess task is that this region is important for spatial processing skills (Marshall et al., 1997; Fink et al., 2000, 2001; Powell et al., 2012b; Richter et al., 1997), and the ability to focus attention on a particular stimuli or objective (Abu-Akel and Shamay-Tsoory, 2011), involved in the game of chess (Atherton et al., 2003; Campitelli et al., 2005).

ToM tasks that require considering the contents of another person's mind (an internally focused process) recruit dorsal PFC (Frith and Frith, 2003; German et al., 2004) and orbital PFC (Powell et al., 2014). Previous research supports the role of BA11 (which resides within orbital PFC and partially within ventrolateral and ventromedial PFC) in both ToM tasks (Lewis et al., 2011) and empathizing tasks (Farrow et al., 2001). Greater activity is observed in ventrolateral PFC and medial PFC when individuals are asked to make empathic judgments relative to other forms of social reasoning (Farrow et al., 2001). The role of this region during empathizing tasks is suggested to include the ability to inhibit one's own experience during the consideration of another's state of mind (Vogeley et al., 2001; Samson et al., 2005). Activation of BA11 was not found in the chess task suggesting that this task required neither empathic judgment nor inhibition of one's own experience when considering the mental state of another.

ToM and chess tasks were associated with significant activation in a further six regions: left hemisphere cuneus [BA18], fusiform gyrus [BA37], inferior frontal gyrus [BA45], and right hemisphere pyramis/cerebellum posterior lobe, lingual gyrus [BA18], and middle occipital gyrus [BA19]. The majority of these regions i.e. BA18, BA19, BA37 and cerebellum were observed in the study by Völlm et al. (2006) in relation to ToM processing. A number of studies have also reported a role for the fusiform gyrus [BA37] in chess tasks (e.g. Righi and Tarr, 2004; Bukach et al., 2006). The suggestion has been that the face-selective fusiform area can be hijacked to process chess patterns; however, the findings regarding the role of the fusiform gyrus in chess processing are inconsistent (Krawczyk et al., 2011). Our findings suggest that the fusiform area might support internal representations at least at an early perceptual level. That BA18 and BA19 within the occipital lobe are involved in the ToM task is in line with some other literature showing a role for occipital cortex in ToM tasks (Enrici et al., 2011).

Areas associated with chess when explored across the whole brain included, left hemisphere precuneus [BA7], caudate tail, precentral gyrus [BA6], middle frontal gyrus [BA6 and BA46], posterior cingulate gyrus [BA23] and culmen/cerebellum anterior lobe and right hemisphere middle frontal gyrus [BA9] and declive/cerebellum posterior lobe. This is consistent with previous studies that have explored the neural correlates of chess (i.e. Atherton et al., 2003; Campitelli et al., 2005). Campitelli et al. (2005) also found distributed neural activation in BA10, BA13, BA22, BA29, BA32, BA38, BA44, BA45, and $B A 47$, although it is worth noting that five of these regions had a cluster size of $k<10$ voxels - the present study considered only clusters of 10 or more voxels. Using a sample of seven novice male chess players Atherton et al. (2003) found activation bilaterally in BA7, BA19,
BA39 and BA40 and left hemisphere BA6, BA8, BA9 and cerebellum. Unlike the studies of Atherton et al. (2003) and Campitelli et al. (2005) that used a one-shot scene of a chess game, the task used in the present study required the participants to follow a series of shots of a game progressing and to decide (using a forced choice task) which out of two options they would make next if they were playing. This task may be considered more cognitively demanding than those previously used.

The present study did not incorporate the three tasks into a single paradigm and therefore did not allow for direct comparisons in neural activation between the different tasks. To directly test for differences in neural activation between ToM- and chess-related tasks, future studies should consider incorporating these two different measures into the same paradigm. The present study used a well-established ToM task and empathizing task (Brunet et al., 2000; Völlm et al., 2006) to activate neural areas associated with either ToM and empathizing. Chess level, in this study was calculated by multiplying the intensity at which the individual has played chess since they began playing the game by their score on the fMRI chess task. Other scales for measuring chess are available, for example the Elo scale (Elo, 1978) which is an international ranking of chess expertise. However, our measure of chess expertise is based on the performance on the task employed in the study which is taken from a database of chess games played by masters and grand masters. Cumulative hours of serious study alone are thought to be the best predictor of a players' current chess rating (Charness et al., 2005). The present study did not include hours of chess study within the calculation of chess expertise due to the age range of this small sample and secondly, because chess players were novice chess players who did not engage in serious study of the game. The fact that only novice chess players were used in the present study could have implications for our results, as neuroimaging results show that chess experts compared to novices present increased neuronal activation in the ventral visual system, TPJ, posterior cingulate gyrus, and orbitofrontal cortex (Atherton et al., 2003; Bilalić et al., 2010, 2011a,b, 2012; Krawczyk et al., 2011; Rennig et al., 2013). Such differences in neuronal activity could be explained by differences in the strategies employed between chess novices and experts when playing the game of chess. Chess players automatically activate chunks (perceptual patterns that can be used as units of meaning) in long-term memory when perceiving familiar positions (Chase and Simon, 1973; Gobet, 1998). At the novice level chess strategies involve reasoning iteratively about the potential moves of the opponent. Future studies should therefore consider testing the neural overlap between ToM processing and the game of chess in a group of expert chess players.

The acquisition of ToM has important consequences as it allows individuals to negotiate through a largely social word, for example, in conversations, negotiations, the development of social relationships, moral judgments and emotions and feeling trust (Korkmaz, 2011). ToM deficits are observed in individuals with a range of neurodevelopmental disorders, such as autism (Happé and Frith, 1996), schizophrenia (Frith, 2014), 

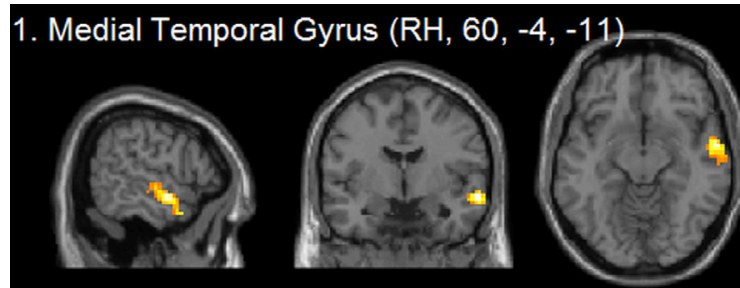

2. Medial Temporal Gyrus (LH, $-60,-16,-8$ )
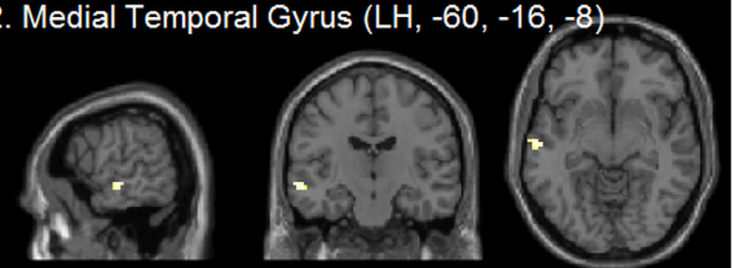

3. Medial Orbitofrontal Cortex (RH, 6, 50,-11)
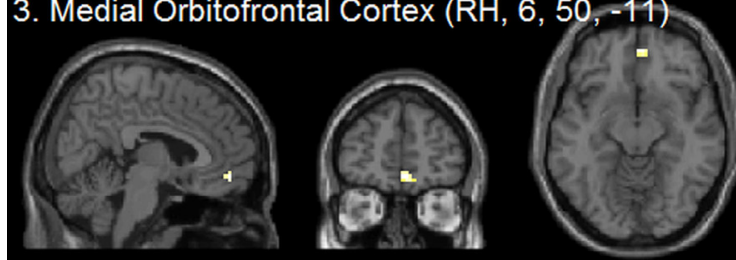

4. Temporoparietal Junction ( $\mathrm{RH}, 51,-52,25)$
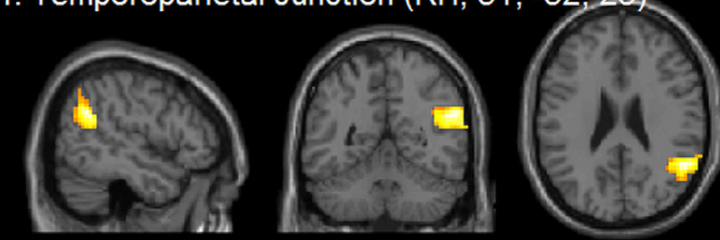

5. Posterior Cingulate Gyrus (LH, $-6,-55,22)$
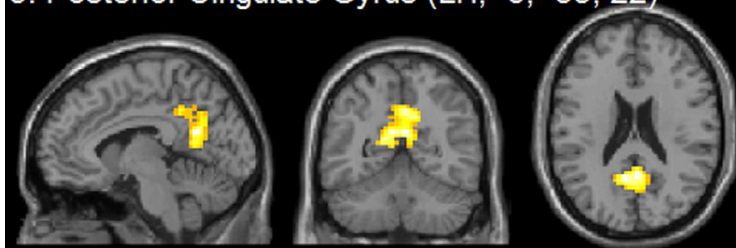

6. Superior Temporal Gyrus (LH, $-69,-52,16)$
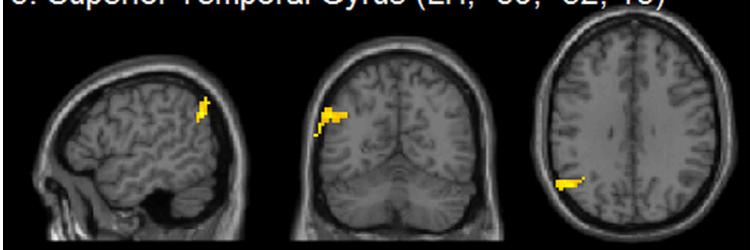

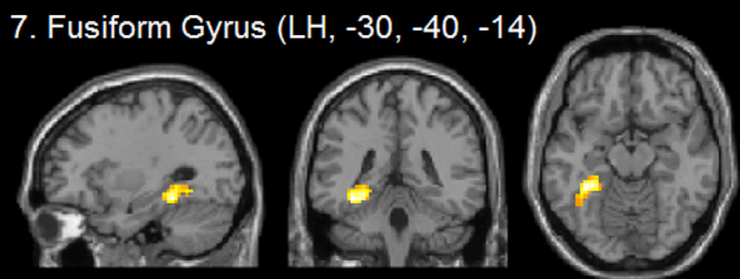

8. Cerebellum Posterior Lobe (RH, 15, -82, -29)
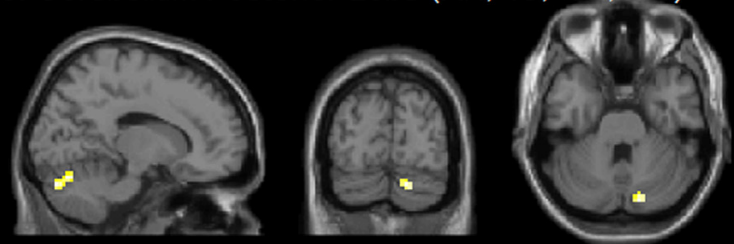

9. Lingual Gyrus $(\mathrm{RH}, 21,-97,-5)$
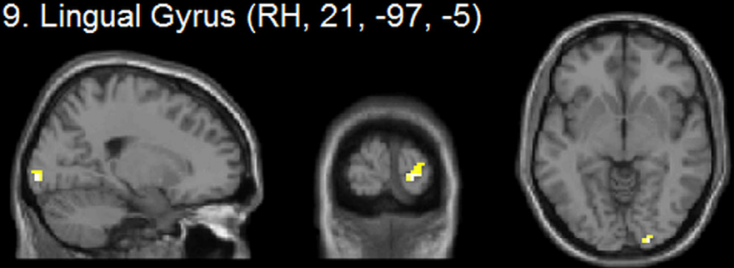

10. Medial Occipital Gyrus (RH, $42,-73,1)$
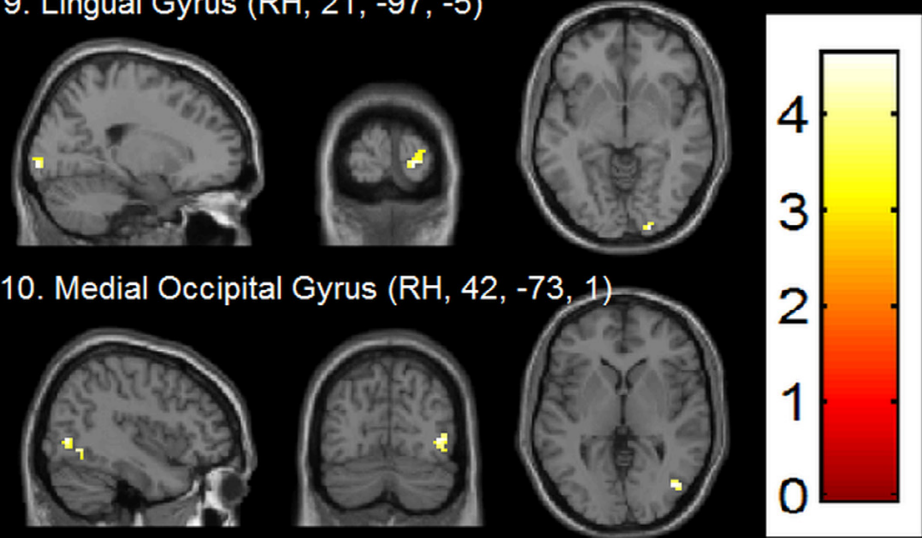

11. Cuneus (LH, $-24,-100,7)$
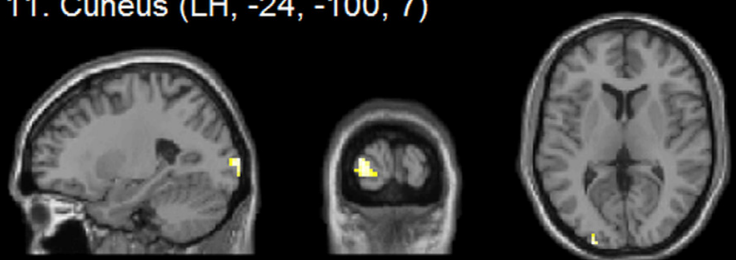

12. Inferior Frontal Gyrus (LH, $-57,23,13)$
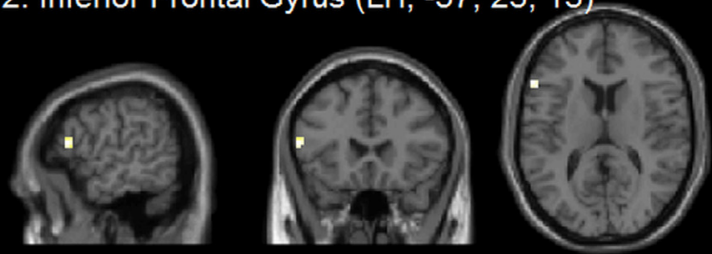

Fig. 3. Twelve areas of activation yielded by the contrast 'ToM'-'Physical 1' (tested across the whole brain) corrected for multiple comparisons $(P<0.05, \mathrm{FDR})$ shown on sagittal, coronal and axial planes on a single subject $\mathrm{T}_{1} \mathrm{MR}$ image supplied by SPM8. Regions were extracted using the toolbox MarsBaR for SPM8. Talairach coordinates $(x, y, z)$ are given for the most significant voxel in each cluster. The corresponding BA region for the Talairach coordinates can be seen in Appendix A. The twelve different regions are numbered. $\mathrm{LH}=\mathrm{left} \mathrm{hemisphere}, \mathrm{RH}=$ right hemisphere.

bipolar affective disorder (Bora et al., 2005), learning difficulty (Capps et al., 1998), attention deficit hyperactivity disorder (Uekermann et al., 2010) as well as Tourette's Syndrome (Eddy et al., 2011). However, not all those with autism fail ToM tests. Findings from the present study may suggest that those with deficits in ToM processing might perform to a high level at chess tasks by depending on chess associated regions outside the internal representational network i.e. they would quickly employ a strategy of activating chunks in long-term memory when faced with a state of play during the game of chess.
A 'core-network' for ToM has been proposed, which includes mPFC and bilateral TPJ (Amodio and Frith, 2006; Frith and Frith, 2006; Mitchell et al., 2002; Schurz et al., 2014). These regions are consistently engaged whenever we reason about the mental states of others, regardless of task and stimuli (Schurz et al., 2014). A large degree of overlap concerning areas of activation for the ToM task and the empathizing task is not surprising given that the selected empathizing task involves cognitively inferring another's affective state rather than resonating with another's affective state (Singer et al., 2004). The 
chess task involved following the game of chess and deciding which out of two moves to make next. We hypothesize that in our cohort of novice chess players the strategy was to employ an iterative thinking pattern which in part involved mentalizing processes and recruited core ToM-related regions. This differs to previous conceptions of chess which is wholly considered as a game of pure strategy.

\section{SUMMARY}

Findings from the present study support the evidence that there is a core network which is recruited during ToM tasks which form the core representational areas, and were recruited during all three tasks in this study, with task-specific areas commonly co-recruited alongside the core areas dependent on the nature of the internal representational task. The present study demonstrates that, at least in novice chess players, there is a large degree of neural overlap when considering potential moves on a chess board and when considering ToM scenarios. Future research should replicate the study using a group of expert chess players. While these results suggest that ToM and chess processing depend on a very similar neural network, ToM also depends on those regions responsible for inhibition of one's own experience when considering the mental state of another and for visual evaluation of action rationality, which are themselves not unique to ToM itself as they are equally observed during tasks requiring empathy, namely frontal gyrus/orbitofrontal cortex and bilateral middle temporal gyrus. Chess, in contrast, depends on an extended network covering middle frontal [BA6 and 46] and parietal cortex [BA7]. Individuals with deficits in ToM processing, such as those with autism, would perform poorly at ToM tasks but might perform to a high level at chess tasks by depending on chess associated regions outside the internal representational network.

Acknowledgements-This work was funded by EPSRC Shaping Capability funds research grant. All work was carried out at the Magnetic Resonance and Image Analysis Research Centre (MARIARC) at the University of Liverpool. We are grateful to Valerie Adams, radiographer at MARIARC, for her assistance in the data. We give our thanks to all those who participated in this study and to the Merseyside Chess clubs for their support, enthusiasm and assistance, throughout this study and during the recruitment of participants.

\section{REFERENCES}

Abu-Akel A, Shamay-Tsoory S (2011) Neuroanatomical and neurochemical bases of theory of mind. Neuropsychologia 49 (11):2976.

Allison T, Puce A, McCarthy G (2000) Social perception from visual cues: role of the STS region. Trends Cogn Sci 4:267-278.

Amodio DM, Frith CD (2006) Meeting of minds: the medial frontal cortex and social cognition. Nat Rev Neurosci 7(4):268-277.

Atherton M, Zhuang J, Bart WM, Hu X, He S (2003) A functional MRI study of high-level cognition. I. The game of chess. Cognitive Brain Res 16:26-31.

Baron-Cohen S, Wheelwright S (2004) The empathy quotient: an investigation of adults with Asperger syndrome or high functioning autism, and normal sex differences. J Autism Dev Disord 34 (2):163-175.

Baron-Cohen S, Wheelwright S, Hill J, Raste Y, Plumb I (2001) The "Reading the mind in the eyes" test revised version: A study with normal adults, and adults with asperger syndrome or highfunctioning autism. J Child Psychol Psyc 42(2):241-251.

Behrens TE, Hunt LT, Rushworth MF (2009) The computation of social behavior. Science 324:1160-1164.

Bernhardt BC, Singer T (2012) The neural basis of empathy. Annu Rev Neurosci 35:1-23.

Bilalić M, Langner R, Erb M, Grodd W (2010) Mechanisms and neural basis of object and pattern recognition: A study with chess experts. J Exp Psychol Gen 139:728-742.

Bilalić M, Kiesel A, Pohl C, Erb M, Grodd W (2011a) It takes twoskilled recognition of objects engages lateral areas in both hemispheres. PLoS One 6:e16202.

Bilalić M, Langner R, Ulrich R, Grodd W (2011b) Many faces of expertise fusiform face area in chess experts and novices. J Neurosci 31:10206-10214.

Bilalić M, Turella L, Campitelli G, Erb M, Grodd W (2012) Expertise modulates the neural basis of context dependent recognition of objects and their relations. Hum Brain Mapp 33:2728-2740.

Bora E, Vahip S, Gonul AS, Akdeniz F, Alkan M, Ogut M, Eryavuz A (2005) Evidence for theory of mind deficits in euthymic patients with bipolar disorder. Acta Psychiat Scand 112:110-116.

Brunet E, Sarfati Y, Hardy-Bayle MC, Decety J (2000) A PET investigation of the attribution of intentions with a nonverbal task. Neuroimage 11:157-166.

Bukach CM, Gauthier I, Tarr MJ (2006) Beyond faces and modularity: the power of an expertise framework. Trends Cogn Sci 10:159-166.

Camerer CF (2009) Behavioural game theory and the neural basis of strategic choice. In: Glimcher PW, Camerer CF, Fehr E, Poldrack RA, editors. Neuroeconomics: decision making and the brain. London: Elsevier. p. 193-206.

Camerer C, Loewenstein G, Prelec D (2005) Neuroeconomics: How neuroscience can inform economics. J Econ Lit 43(1):9-64.

Campitelli G, Gobet F, Parker A (2005) Structure and stimulus familiarity: A study of memory in chess-players with functional magnetic resonance imaging. Span J Psychol 8:238-245.

Capps L, Kehres J, Sigman M (1998) Conversational abilities among children with autism and children with developmental delays. Autism 2:325-344.

Carrington SJ, Bailey AJ (2009) Are there Theory of Mind regions in the brain? A review of the neuroimaging literature. Hum Brain Mapp 30:2313-2335.

Charness N, Tuffiash M, Krampe R, Reingold E, Vasyukova E (2005) The role of deliberate practice in chess expertise. Appl Cogn Psychol 19(2):151-165.

Chase WG, Simon HA (1973) Perception in chess. Cogn Psychol 4:55-81.

De Groot A, Gobet F (1996) Perception and memory in chess. Assen, Holland: Van Gorcum.

Eddy CM, Mitchell IJ, Beck SR, Cavanna AE, Rickards H (2011) Social reasoning in Tourette syndrome. Cogn Neuropsychiatry 16 (4):326-347.

Elo A (1978) The rating of chessplayers, past and present. New Arco.

Enrici I, Adenzato M, Cappa S, Bara BG, Tettamanti M (2011) Intention processing in communication: A common brain network for language and gestures. J Cogn Neurosci 23(9):2415-2431.

Farrow TFD, Zheng Y, Wilkinson ID, Spence SA, Deakin JFW, et al. (2001) Investigating the functional anatomy of empathy and forgiveness. Neuroreport 12:2849-2853.

Fink GR, Marshall JC, Shah NJ, Weiss PH, Halligan PW, GrosseRuyken M, et al. (2000) Line bisection judgments implicate right parietal cortex and cerebellum as assessed by fMRI. Neurology 54(6):1324-1331

Fink GR, Marshall JC, Weiss PH, Zilles K (2001) The neural basis of vertical and horizontal line bisection judgments: an fMRI study of normal volunteers. Neuroimage 14(1):S59-S67. 
Friston KJ, Ashburner J, Frith CD, Poline JB, Heather JD, Frackowiak RSJ (1995a) Spatial registration and normalization of images. Hum Brain Mapp 3(3):165-189.

Friston KJ, Holmes AP, Poline JB, Grasby PJ, Williams SCR, Frackowiak RSJ, et al. (1995b) Analysis of $\mathrm{fMRI}$ time-series revisited. Neuroimage 2(1):45-53.

Friston KJ, Jezzard P, Turner R (1994) Analysis of functional MR time-series. Hum Brain Mapp 1(2):153-171.

Frith CD (2014) The cognitive neuropsychology of schizophrenia. Hove, UK: Psychology Press.

Frith CD, Frith U (1999) Interacting minds: a biological basis. Science 286(5445):1692-1695.

Frith CD, Frith $U$ (2006) The neural basis of mentalizing. Neuron 50 (4):531-534.

Frith U, Frith CD (2003) Development and neurophysiology of mentalizing. Philos T Roy Soc B 358(1431):459-473.

Gallagher HL, Happe F, Brunswick N, Fletcher PC, Frith U, Frith CD (2000) Reading the mind in cartoons and stories: an fMRI study of "theory of mind" in verbal and nonverbal tasks. Neuropsychologia 38:11-21.

Gallagher HL, Jack AI, Poepstorff A, Frith CD (2002) Imaging the intentional stance in a competitive game. Neuroimage 16:814-821.

Gallese V (2003) The roots of empathy: the shared manifold hypothesis and the neural basis of intersubjectivity. Psychopathology 36:171-180.

German TP, Niehaus JL, Roarty MP, Giesbrecht B, Miller MB (2004) Neural correlates of detecting pretense: automatic engagement of the intentional stance under covert conditions. J Cogn Neurosci 16:1805-1817.

Gobet F (1997) A pattern-recognition theory of search in expert problem solving. Think Reasoning 3:291-313.

Gobet F (1998) Chess players' thinking revisited. Swiss J Psychol 57:18-32.

Gobbini MI, Koralek AC, Bryan RE, Montgomery KJ, Haxby JV (2007) Two takes on the social brain: a comparison of theory of mind tasks. J Cogn Neurosci 19(11):1803-1814.

Happé F, Frith U (1996) The neuropsychology of autism. Brain 119:1377-1400.

Jastorff J, Orban GA (2009) Human functional magnetic resonance imaging reveals separation and integration of shape and motion cues in biological motion processing. J Neurosci 29:7315-7329.

Jastorff J, Clavagnier S, Gergely György, Orban GA (2011) Neural mechanisms of understanding rational actions: middle temporal gyrus activation by contextual violation. Cereb Cortex 21:318-329.

King-Casas B, Sharp C, Lomax-Bream L, Lohrenz T, Fonagy P, et al. (2008) The rupture and repair of cooperation in borderline personality disorder. Science 321:806-810.

Korkmaz B (2011) Theory of mind and neurodevelopmental disorders of childhood. Pediatr Res 69:101R-108R.

Krawczyk DC, Boggan AL, McClelland MM, Bartlett JC (2011) The neural organization of perception in chess experts. Neurosci Lett 499:64-69.

Lewis PA, Rezaie R, Brown R, Roberts N, Dunbar RIM (2011) Ventromedial prefrontal volume predicts understanding of others and social network size. Neuroimage 57(4):1624-1629.

Lawrence EJ, Shaw P, Giampietro VP, Surguladze S, Brammer MJ, David AS (2006) The role of 'shared representations' in social perception and empathy: an fMRI study. Neuroimage 29:1173-1184.

Lieberman MD (2007) Social cognitive neuroscience: A review of core processes. Annu Rev Psychol 58:259-289.

Maldjian JA, Laurienti PJ, Burdette JH (2004) Precentral gyrus discrepancy in electronic versions of the talairach Atlas. Neuroimage 21(1):450-455.

Marshall RS, Lazar RM, Van Heertum RL, Esser PD, Perera GM, Mohr JP (1997) Changes in regional cerebral blood flow related to line bisection discrimination and visual attention using HMPAOSPECT. Neuroimage 6(2):139-144.
McCabe K, Houser D, Ryan L, Smith V, Trouard T (2001) A functional imaging study of cooperation in two-person reciprocal exchange. Proc Natl Acad Sci U S A 98(20):11832-11835.

Mitchell JP, Heatherton TF, Macrae CN (2002) Distinct neural systems subserve person and object knowledge. Proc Natl Acad Sci U S A 99(23):15238-15243.

Mitchell JP (2009) Inferences about mental states. Phil T Roy Soc B 364:1309-1316.

Powell JL, Lewis PA, Dunbar RIM, García-Fiñana M, Roberts N (2010) Orbital prefrontal cortex volume correlates with social cognitive competence. Neuropsychologia 48:3554-3562.

Powell JL, Lewis P, Roberts N, García-Fiñana M, Dunbar R (2012a) Orbital prefrontal cortex volume predicts social network size: an imaging study of individual differences in humans. Proc R Soc B Biol Sci 279(1736):2157-2162.

Powell JL, Kemp G, García-Fiñana M (2012b) Association between language and spatial laterality and cognitive ability: An fMRI study. Neuroimage 59:1818-1829.

Powell JL, Kemp G, Dunbar RIM, Sluming V, Roberts N, GarcíaFiñana M (2014) Association between intentionality competence and prefrontal volume in left- and right-handers. Cortex 54:63-76.

Rennig J, Bilalic M, Huberle E, Karnath HO, Himmelbach M (2013) The temporo-parietal junction contributes to global gestalt perception: Evidence from studies in chess experts. Front Hum Neurosci 7(513):1-11.

Richter W, Ugurbil K, Georgopoulos A, Kim S (1997) Time-resolved fMRI of mental rotation. Neuroreport 8:3697-3702.

Righi G, Tarr MJ (2004) Are chess experts any different from face, bird, or Greeble experts? J Vision 4:504a.

Sally D, Hill E (2006) The development of interpersonal strategy: autism, theory-of-mind, cooperation and fairness. J Econ Psychol 27(1):73-97.

Samson D, Apperly IA, Kathirgamanathan U, Humphreys GW (2005) Seeing it my way: a case of selective deficit in inhibiting selfperspective. Brain 128:1102-1111.

Saxe R, Carey S, Kanwisher N (2004) Understanding other minds: linking developmental psychology and functional neuroimaging. Annu Rev Psychol 55:87-124.

Saxe R, Kanwisher N (2003) People thinking about thinking people. The role of the temporo-parietal junction in "theory of mind". Neuroimage 19(4):1835-1842.

Saxe R, Wexler A (2005) Making sense of another mind: The role of the right temporo-parietal junction. Neuropsychologia 43:1391-1399.

Schurz M, Radua J, Aichhorn M, Richlan F, Perner J (2014) Fractionating theory of mind: A meta-analysis of functional brain imaging studies. Neurosci Biobehav Rev 42C:9-34.

Singer T, Seymour B, O'Doherty J, Kaube H, Dolan RJ, Frith CD (2004) Empathy for pain involves the affective but not sensory components of pain. Science 303:1157-1162.

Spreng RN, Mar RA, Kim ASN (2009) The common neural basis of autobiographical memory, prospection, navigation, Theory of Mind, and the default mode: A quantitative meta-analysis. J Cogn Neurosci 21(3):489-510.

Stiller J, Dunbar RIM (2007) Perspective-taking and memory capacity predict social network size. Soc Networks 29:93-104.

Tayama M, Tateno M, Park TW, Ukai W, Hashimoto E, Saito T (2012) The study of cognitive characteristics in Asperger's disorder by using a modified prisoner's dilemma game with a variable payoff matrix. PLoS One 7(11):e48794.

Uekermann J, Kraemer M, Abdel-Hamid M, Schimmelmann BG, Hebebrand J, Daum I, Wiltfang J, Kis B (2010) Social cognition in attention-deficit hyperactivity disorder (ADHD). Neurosci Biobehav Rev 34:734-743.

Vogeley K, Bussfeld P, Newen A, Herrmann S, Happé F, Falkai P, et al. (2001) Mind reading: neural mechanisms of theory of mind and self-perspective. Neuroimage 14(1):170-181.

Völlm BA, Taylor ANW, Richardson P, Corcoran R, Stirling J, McKie S, Deakin JFW, Ellitt R (2006) Neuronal correlates of theory of mind and empathy: A functional magnetic resonance imaging study in a nonverbal task. Neuroimage 29:90-98. 


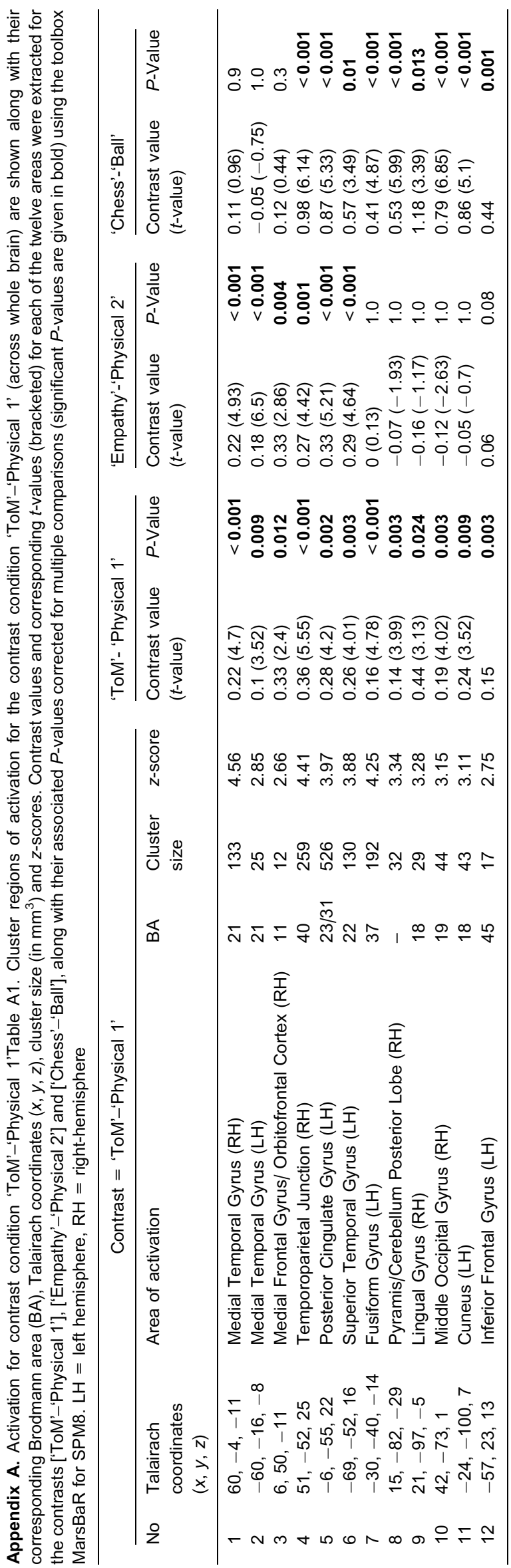


Appendix B. Activation for contrast conditions 'Empathy'-'Physical 2' and 'Chess'-'Ball' Table B1. Cluster regions of activation for the contrast conditions 'Empathy'-'Physical 2' and 'Chess'-'Ball' tested across the whole brain are shown along with their corresponding Brodmann area (BA), Talairach coordinates $(x, y, z)$, cluster size (in $\mathrm{mm}^{3}$ ) and $z$-scores. $\mathrm{LH}=$ left hemisphere, $\mathrm{RH}=$ right-hemisphere

\begin{tabular}{|c|c|c|c|c|}
\hline Talairach coordinates $(x, y, z)$ & Area of activation & BA & Cluster size & z-score \\
\hline \multicolumn{5}{|c|}{ Contrast $=$ 'Empathy' - 'Physical 2' } \\
\hline $12,-52,34$ & Precuneus (RH) & 7 & 1048 & 5.71 \\
\hline $57,-13,-5$ & Medial Temporal Gyrus (RH) & 21 & 274 & 5.34 \\
\hline$-60,-10,-14$ & Medial Temporal Gyrus (LH) & 21 & 966 & 5.18 \\
\hline $54,-52,28$ & Temporoparietal Junction (RH) & 40 & 193 & 4.96 \\
\hline$-1,-19,34$ & Cingulate Gyrus (LH) & 24 & 114 & 4.48 \\
\hline$-3,53,31$ & Superior Frontal Gyrus (LH) & 9 & 751 & 4.37 \\
\hline $3,26,10$ & Anterior Cingulate Gyrus (RH) & 24 & 94 & 4.08 \\
\hline$-54,26,1$ & Inferior Frontal Gyrus (LH) & 45 & 35 & 3.88 \\
\hline$-6,17,-5$ & Anterior Cingulate Gyrus (LH) & 24 & 57 & 3.82 \\
\hline$-27,-79,-26$ & Uvula/Cerebellum Posterior Lobe (LH) & - & 39 & 3.64 \\
\hline $3,50,-11$ & Medial Frontal Gyrus/Orbitofrontal Cortex (RH) & 11 & 123 & 3.49 \\
\hline $48,-31,31$ & Inferior Parietal Lobule $(\mathrm{RH})$ & 40 & 57 & 3.13 \\
\hline \multicolumn{5}{|c|}{ Contrast $=$ 'Chess' - 'Ball' } \\
\hline $30,-61,-23$ & Declive/Cerebellum Posterior Lobe (RH) & - & 2391 & 6.56 \\
\hline$-21,-73,46$ & Precuneus (LH) & 7 & 1008 & 6.40 \\
\hline $57,-52,28$ & Temporoparietal Junction (RH) & 40 & 99 & 5.58 \\
\hline$-18,-31,16$ & Caudate (LH) & Caudate tail & 48 & 5.17 \\
\hline$-48,-1,37$ & Precentral Gyrus (LH) & 6 & 197 & 5.08 \\
\hline$-45,26,25$ & Middle Frontal Gyrus (LH) & 46 & 156 & 4.88 \\
\hline$-33,-4,55$ & Middle Frontal Gyrus (LH) & 6 & 69 & 4.84 \\
\hline$-30,-55,-26$ & Culmen/Cerebellum Anterior Lobe (LH) & - & 68 & 4.81 \\
\hline$-66,-52,19$ & Posterior Cingulate Gyrus (LH) & 23 & 22 & 4.68 \\
\hline $45,38,28$ & Middle Frontal Gyrus (RH) & 9 & 69 & 4.58 \\
\hline
\end{tabular}

